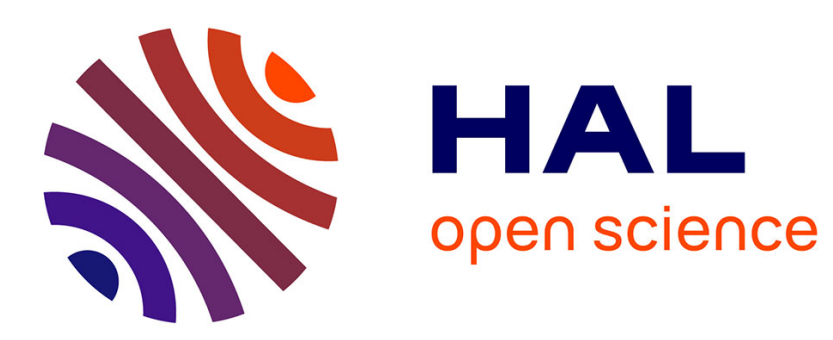

\title{
Comparison of Blur Edge Time and Gaussian Edge Time as measures of motion blur
}

Sylvain Tourancheau, Kjell Brunnström, Andrew B. Watson, Borje Andrén

\section{To cite this version:}

Sylvain Tourancheau, Kjell Brunnström, Andrew B. Watson, Borje Andrén. Comparison of Blur Edge Time and Gaussian Edge Time as measures of motion blur. SID International Symposium, Seminar and Exhibition, May 2010, Seattle, United States. pp.14.1. hal-00486938

\section{HAL Id: hal-00486938 \\ https://hal.science/hal-00486938}

Submitted on 27 May 2010

HAL is a multi-disciplinary open access archive for the deposit and dissemination of scientific research documents, whether they are published or not. The documents may come from teaching and research institutions in France or abroad, or from public or private research centers.
L'archive ouverte pluridisciplinaire HAL, est destinée au dépôt et à la diffusion de documents scientifiques de niveau recherche, publiés ou non, émanant des établissements d'enseignement et de recherche français ou étrangers, des laboratoires publics ou privés. 


\title{
14.1: Comparison of Blur Edge Time and Gaussian Edge Time as measures of motion blur
}

\author{
Sylvain Tourancheau ${ }^{1}$, Kjell Brunnström ${ }^{2}$, Andrew B. Watson ${ }^{3}$ and Börje Andrén ${ }^{2}$ \\ 1 IRCCyN, Polytech'Nantes, University of Nantes, 44300 Nantes, France \\ 2 NetLab: IPTV, Video and Display Quality, Acreo AB, SE-16440 Kista, Sweden \\ ${ }^{3}$ MS 262-2 NASA Ames Research Center, Moffett Field, CA 94035-1000 \\ Corresponding author: kjell.brunnstrom@acreo.se
}

\begin{abstract}
Blur Edge Time has been shown to be a reasonable metric for characterisation of motion blur of LCD displays. It can be estimated by taking the 10\% to 90\% level of the Moving Edge Temporal Profile or by using the standard deviation of a fitted cumulative Gaussian function. In this paper we will compare these two ways of estimating the Blur Edge Time. Ultimately the usefulness of these metrics of motion blur is whether they are good predictors of perceived motion blur.
\end{abstract}

\section{Introduction}

Display motion blur is a perceptual phenomenon that is the result of the interaction between the temporal update of a pixel and the visual tracking by the human visual system of a moving object. The visual experience of a moving sharp edge is that it becomes visually broader. It has been shown that this broadening of the edge is linearly dependent on the speed of the edge [1,2]. We refer to the cross-section of a moving edge as the Moving Edge Spatial Profile (MESP). A metric of the width of the edge, called Blur Edge Width $(B E W)$, can be defined as the distance between the $10 \%$ to $90 \%$ level of the profile.

To measure this width directly, a tracking camera that is either moved along the moving edge or utilizing rotating mirrors, or a stationary high speed camera can be used[3-5]. Since it is assumed that $B E W$ is linearly dependent on the speed, we generally consider what we call the Moving Edge Temporal Profile (METP) by scaling the MESP with speed. It has been shown $[5,6,7]$ that METP can be derived from the temporal stepresponse of the display pixels by convolving it with a rectangular pulse of one frame time. This permits to directly use non-imaging device such as photometers to measure the METP.
From this METP an analogous metric to $B E W$ can be defined called the Blur Edge Time (BET) i.e. by taking the time between the $10 \%$ and $90 \%$ level of METP. As expected the relationship between $B E W$ and $B E T$ is $B E W=B E T \cdot V$. Watson (2009) [8] proposed another metric that consists of fitting a cumulative Gaussian function to the METP. Then the time interval from $10 \%$ to $90 \%$ of the METP can be estimated from the standard deviation $\sigma$ of the cumulative Gaussian function. This metric was named Gaussian Edge Time (GET).

This paper will compare BET and GET metrics with each other, on 12 various displays and for 20 different gray-to-gray transitions. For each display and each transition, METP has been measured with a non-imaging device, and only this method has been used here. We will particularly focus on the reproducibility of the estimates and on the correlation between both metrics. Another purpose is to see how well they can predict the results of a user experience evaluation described previously [1].

\section{Methodology}

\subsection{Displays under test}

Displays that have been assessed in this work are described in Table 1. All of them are liquid-crystal displays (LCD). They were equipped with various features that are described in Table 1 .

\subsection{Temporal step response measurements}

On each DUT, temporal step-responses of the pixels have been measured for 20 transitions from one gray level $\left(g_{\text {start }}\right)$ to another $\left(g_{\text {end }}\right)$ among five. The following gray levels were used: 0, 63, 127, 191, and 255. Each of the 20 gray-to-gray transitions $g_{\text {start }} \rightarrow g_{\text {end }}$ have been measured 5 times on each DUT, except for DUT3, DUT11 and DUT12 (only twice).

Table 1: Displays under test.

\begin{tabular}{|c|c|c|c|l|}
\hline DUTID & Size & Resolution & Type & \multicolumn{1}{|c|}{ Note } \\
\hline DUT1 & $20 "$ & $1680 \times 1050$ & Desktop LCD monitor & \\
\hline DUT2 & $24.1 "$ & $1920 \times 1200$ & Desktop LCD monitor & Strong backlight modulations \\
\hline DUT3 & $42 "$ & $1360 \times 768$ & LCDTV & LED backlight, local dimming turned off \\
\hline DUT4 & $19 "$ & $1280 \times 1024$ & Desktop LCD monitor & \\
\hline DUT5 & $15 "$ & $1024 \times 768$ & Desktop LCD monitor & \\
\hline DUT6 & $24 ”$ & $1920 \times 1200$ & Desktop LCD monitor & Strong backlight modulations \\
\hline DUT7 & $26 "$ & $1920 \times 1200$ & Desktop LCD monitor & Scrolling backlight, Overdrive \\
\hline DUT8 & $20.1 ”$ & $1600 \times 1200$ & Desktop LCD monitor & LED edge backlight \\
\hline DUT9 & $40 "$ & $1920 \times 1080$ & LCDTV & LED backlight, local dimming turned off \\
\hline DUT10 & $30 "$ & $2560 \times 1600$ & Desktop LCD monitor & Scrolling backlight, Overdrive \\
\hline DUT11 & $23 "$ & $1920 \times 1200$ & Desktop LCD monitor & \\
\hline DUT12 & $37 ”$ & $1920 \times 1080$ & LCDTV & \\
\hline
\end{tabular}


The light intensity emitted by the display was read by a photodiode positioned in close contact with the screen surface. The photodiode was surrounded by black velvet in order to reduce any scratches to the display surface and to shield any ambient light reaching the photodiode. The photodiode (Burr-Brown OPT101 monolithic photodiode with on chip transimpedance amplifier) has a fast response ( $28 \mu$ s from $10 \%$ to $90 \%$, rise or fall time). The signal was read by an USB oscilloscope EasyScope II DS1M12 "Stingray" 2+1 Channel PC Digital Oscilloscope/Logger from USB instruments. The accuracy of the instrument has been tested with an LED light source connected to a function generator.

Each transition has been measured sequentially by displaying each gray level during 20 frames, with a sampling period of $0.1 \mathrm{msec}$.

\subsection{Moving edge temporal Profile}

From these temporal step-responses, METP was then computed by convolving the waveform with a rectangular pulse of one frame time, in the same way as described in Tourancheau (2009)[5]. We then trimmed the METP from 15 frames before the transition to 15 frames after. This ensures that this 30 -frame long waveform was clean of any residuals from the convolution or from the previous and next transitions.

\section{Blur estimates}

\subsection{BET metric}

Blurred Edge Time (BET) is usually measured on the METP as the time interval from $10 \%$ to $90 \%$ of the METP luminance range. In order to determine $10 \%$ and $90 \%$ values of the METP, we need to estimate precisely the beginning relative luminance value $B$ and the ending relative luminance value $E$. This has been done by computing the average luminance value of samples corresponding to the first two frames and to the last two frames (respectively) of the METP.

Since some ripples can remain on METP after the convolution, it could have been necessary to apply some additional processing in order to determine for which samples it crossed the $10 \%$ and $90 \%$ values. This was done using a filtering with a Gaussian kernel with a standard deviation of 0.15 frames. An example of METP, with smoothed waveform is shown in Figure 1.

\subsection{GET metric}

The Gaussian Edge Time (GET) were measured according to Watson (2010)[8]. The following cumulative Gaussian function was fitted to the METP:

$$
\begin{aligned}
& G(t)=B+(E-B) \int_{-\infty}^{t} \frac{1}{\sigma \sqrt{2 \pi}} \exp \left(-\frac{(x-\mu)^{2}}{2 \sigma^{2}}\right) d x \\
& G(t)=E+\frac{B-E}{2} \operatorname{erfc}\left(\frac{t-\mu}{\sigma \sqrt{2}}\right)
\end{aligned}
$$

where $E$ and $B$ are beginning and ending relative luminance values, $t$ is the time in seconds, $\mu$ is the mean and $\sigma$ is the standard deviation of the Gaussian, and erfc is the complementary error function. The parameter $\sigma$ can be converted to an estimate of $B E T$ that is referred to as the Gaussian Edge Time (GET) by:

$$
G E T=2.563 \sigma
$$

For additional accuracy, the fitting was done twice. First the parameters were estimated from the complete waveform. Then the waveform was trimmed to the mean $\mu$ plus and minus a number $N_{\sigma}$ of standard deviations $\sigma$, and the fitting was repeated. Various values of $N_{\sigma}$ have been tested here, and the consistency between $B E T$ and $G E T$ has been studied regarding this parameter. An example of METP, with cumulative Gaussian function is shown in Figure 2.

\subsection{Overdrive}

Overdrive techniques to reduce liquid crystal cells response time can lead to overshoot or undershoot on the temporal stepresponses, as well as on the METP. These artifacts are usually taken in account by measuring BET from $-10 \%$ to $110 \%$ if these values are reached [9]. Since GET metric cannot reflect these particular distortions, we computed BET from $10 \%$ to $90 \%$ even in presence of overdrive, in order to compare both metrics equally.

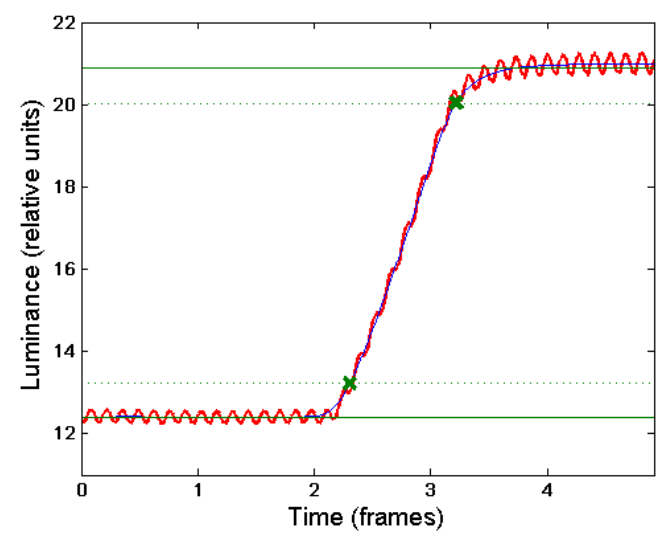

Figure 1: Example of BET estimate for DUT10 and transition 191-255. Thick line (red) represents raw METP waveform, thin line (blue) is the smoothed METP waveform. Horizontal lines (green) figure the beginning and ending relative luminance values as well as the $10 \%$ and $90 \%$ levels. Samples from which $B E T$ is computed are marked with a cross.

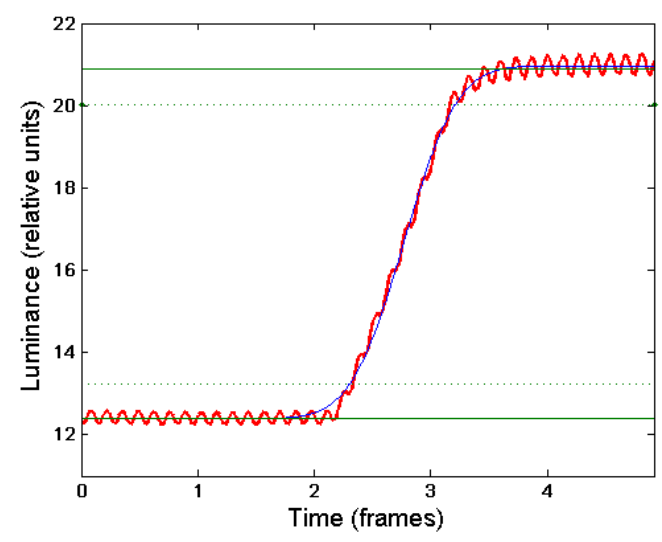

Figure 2: Idem as Figure 1, GET estimate is obtained from the standard deviation of the cumulative Gaussian function obtained from the fitting (thin blue line). 

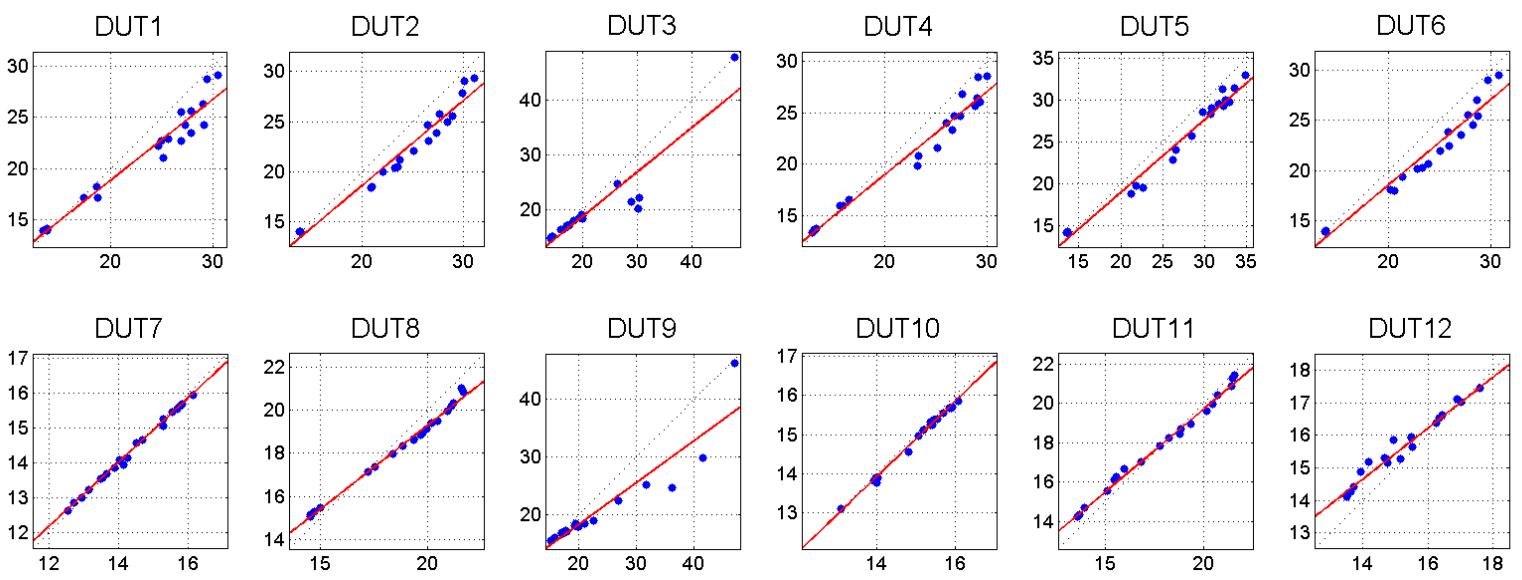

Figure 3: Scatter plot of GET as a function of BET for each DUT.

\section{Results}

\subsection{Correspondence between BET and GET}

We compared the correspondence between BET and GET according to the size of the METP waveform used during the second fitting to determine GET. As explained in Section 3.2, a second fitting was performed to increase the accuracy of the parameters. This second fitting was done on a trimmed METP waveform, from $\mu-N_{\sigma} \cdot \sigma$ to $\mu+N_{\sigma} \cdot \sigma$, with $\mu$ and $\sigma$ the parameters determined by the first fitting. The size of the METP waveform used for the second fitting was, therefore, $2 N_{\sigma} \cdot \sigma$.

Several values of $N_{\sigma}$ were tested: $3,4,5,7$, and 10 , and the linear correlation coefficient between $B E T$ and $G E T$ were computed for each case. Results are presented in Figure 4. It can be observed that the linear correlation coefficient became better as the size of the waveform were increased. Note that when $N_{\sigma}$ were higher than 6, all correlation coefficients were higher than 0.96 , except for DUT3 and DUT9 which are both LCDTVs with LED backlight.

As a conclusion, if we want $G E T$ to be a good predictor of $B E T$, it is necessary to fit the cumulative Gaussian function on a METP waveform which is large enough. If not, some discrepancies can appear between both metrics due to a bad estimation of beginning and ending relative luminance values in the GET computation. In the following, we present a comparison between $B E T$ and $G E T$ with a value of $N_{\sigma}$ fixed to 10 .

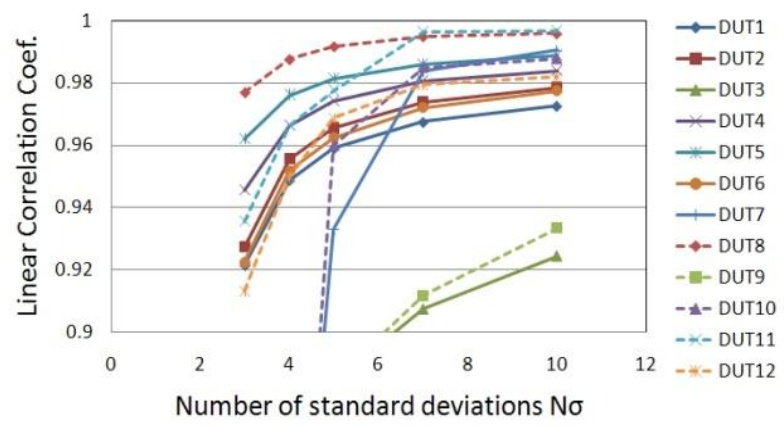

Figure 4: Evolution of the linear correlation coefficient between BET and GET as a function of the size of the METP waveform used to determine GET.
In Figure 5 the correspondence between the two metrics BET and $G E T$ is presented for each display. Table 2 presents the linear correlation coefficient (LCC) and the root-mean-square error (RMSE) between BET and GET over all measurements and for each display. The linear relation $G E T=a B E T+b$ is drawn by a red line in Figure 3 and the values of $a$ and $b$ are given in Table 2 . On all DUT, the LCC between GET and BET is 0.969 and the corresponding linear relation is (cf. Figure 5):

$$
G E T=0.79 \cdot B E T+3.06
$$

Table 2: Linear correlation and root-mean-square error between BET and GET for all DUT.

\begin{tabular}{|c|c|c|c|c|}
\hline ID & LCC & $\mathrm{a}$ & $\mathrm{b}$ & RMSE \\
\hline DUT1 & 0.972 & 0.79 & 3.05 & 2.51 \\
\hline DUT2 & 0.978 & 0.85 & 1.61 & 2.34 \\
\hline DUT3 & 0.924 & 0.81 & 2.53 & 3.43 \\
\hline DUT4 & 0.984 & 0.81 & 2.73 & 2.12 \\
\hline DUT5 & 0.989 & 0.87 & 1.54 & 2.20 \\
\hline DUT6 & 0.977 & 0.85 & 1.53 & 2.41 \\
\hline DUT7 & 0.990 & 0.92 & 1.14 & 0.12 \\
\hline DUT8 & 0.996 & 0.78 & 3.68 & 0.70 \\
\hline DUT9 & 0.933 & 0.73 & 3.60 & 4.26 \\
\hline DUT10 & 0.984 & 0.96 & 0.48 & 0.16 \\
\hline DUT11 & 0.997 & 0.84 & 2.88 & 0.49 \\
\hline DUT12 & 0.982 & 0.78 & 3.73 & 0.55 \\
\hline
\end{tabular}

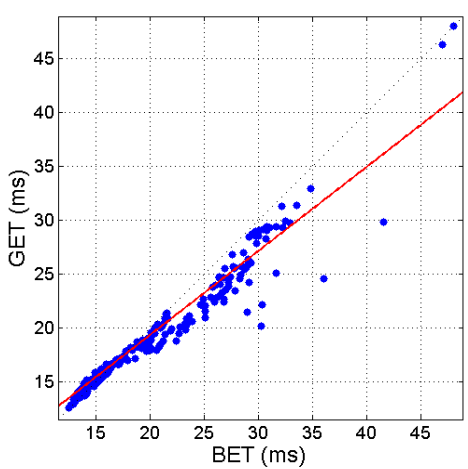

Figure 5: Scatter plot of GET vs BET for all DUT. 


\subsection{Reproducibility of Measurements}

To evaluate the constancy and the reproducibility of measurements, we computed the standard deviation of the set of five measurements for each tested transition. The average of these standard deviation values over the 20 transitions is given for each display in Table 3, except for DUT3, DUT11 and DUT12 which have been measured only twice.

Globally, the average standard deviation between similar measurements is 0.17 for $B E T$, and 0.09 for $G E T$. We can observe from Table 3 that $G E T$ gives more stable results than BET. On DUT with strong backlight modulations such as DUT2 or DUT10, the METP waveform needs to be filtered to measure BET. This leads to high standard deviation values for $B E T$ since the position of backlight modulations regarding the frame refresh can be different from one measurement to another. In spite of this, GET estimates are particularly stable for these DUT.

Table 3: Average standard deviation of measurements for each DUT and for both metrics

\begin{tabular}{|c|c|c|}
\hline ID & BET & GET \\
\hline DUT1 & 0.04 & 0.05 \\
\hline DUT2 & 0.47 & 0.08 \\
\hline DUT4 & 0.12 & 0.11 \\
\hline DUT5 & 0.19 & 0.14 \\
\hline DUT6 & 0.14 & 0.06 \\
\hline DUT7 & 0.13 & 0.06 \\
\hline DUT8 & 0.08 & 0.06 \\
\hline DUT9 & 0.16 & 0.1 \\
\hline DUT10 & 0.22 & 0.11 \\
\hline
\end{tabular}

\subsection{Comparison with user experience data}

In this section we compared the metrics with the results of a user experience described previously [1]. In this subjective experiment users were asked to adjust the blur width of a simulated blurred edge until it matched the motion blur they perceived on a moving edge. From the responses of the observers a Mean Opinion BET (MOBET) were computed, as a subjective measure of the perceived blur in the used displays. This experiment has been led on 3 DUT: DUT10, DUT11 and DUT12.

If we observe these metrics' correspondence to user experience, the fit is reasonable for such a simple metric with a correlation between $B E T$ and $M O B E T$ of 0.785 (RMSE $=2.05$ ) and between GET and MOBET of 0.780 (RMSE = 2.13). Not surprising considering the close correspondence between the metrics, both BET and GET have similar fits and it cannot be said that one is better than the other based on this. It is also unclear whether a higher correlation is possible, given the variability among observers.

\section{Conclusion}

In this paper, we have investigated the relation between the metrics for motion blur BET and GET. Based on the measurements performed on 12 displays and 20 transitions each, $B E T$ and GET are very similar with a 0.97 correlation. We have shown that if we want GET to be a good predictor of $B E T$ it is necessary to estimate it on a waveform with a large number of samples. However, there is no unique method of estimating BET . For example, result will vary depending on the estimation of beginning and ending luminance values, and on the value of the filter standard deviation when filtering is necessary. From this point of view, GET metric is easier to standardize and permits to obtain similar results from one lab to another since there is no unknown parameters.

Despite the high correlation between $B E T$ and $G E T$, relation between them is not identity. We have observed some discrepancies from one display to another but in a whole we obtained $G E T=0.79 \cdot B E T+3.06$.

Finally, both metrics provide a reasonable prediction of the mean opinion of observers, with a correlation is of 0.79 . However, further research is required to build a metric able to predict user experience with accuracy.

\section{Acknowledgements}

This work was partly funded by VINNOVA (The Swedish Governmental Agency for Innovation Systems) which is hereby gratefully acknowledged. ABW was supported in part by NASA's Space Human Factors Engineering Project, WBS 466199.

\section{References}

[1] Tourancheau, S., Le Callet, P., Brunnström, K., and Andrén, B., "Psychophysical study of LCD motion-blur perception", Proc. of SPIE-IS\&T Human Vision and Electronic Imaging XII, 7240, B. Rogowitz and T. N. Pappas Eds., paper 51 (2009)

[2] Teunissen, K., Zhang, Y., Li, X., and Heynderickx, I., "Method for predicting motion artifacts in matrix displays", Journal of the Society for Information Display 14, 957-964 (2006)

[3] Oka, K. and Enami, Y., "43.4: Moving Picture Response Time (MPRT) Measurement System", SID Symposium Digest of Technical Papers 35(1), 1266-1269 (2004)

[4] Someya, J., "19.3: Correlation between Perceived Motion Blur and MPRT Measurement", SID Symposium Digest of Technical Papers 36(1), 1018-1021 (2004)

[5] Tourancheau, S., Brunnström, K., Andrén, B., and Le Callet, P., "LCD motion-blur estimation using different measurement methods", Journal of the Society for Information Display 17, 239. 249 (2009)

[6] Feng, X., Pan, H., and Daly, S., "Comparisons of motion-blur assessment strategies for newly emergent LCD and backlight driving technologies", Journal of the Society for Information Display 16, 981-988 (2008)

[7] Watson, A. B., "31.1: Invited Paper: The Spatial Standard Observer: A Human Vision Model for Display Inspection", SID Symposium Digest of Technical Papers 37(1), 1312-1315 (2006)

[8] Watson, A. B., "Display motion blur: Comparison of measurement methods." Journal of the Society for Information Display 18(2): 179-190 (2010).

[9] VESA, "Flat Panel Display Measurements", Tech. Rep. Version 2.0, Video Electronics Standards Association, 2005. 